\title{
Violencia de Género en Estudiantes Universitarias Madres de la Sede de Occidente- UCR, durante el periodo 2011-2012
}

\author{
Gender-based Violence in mothers who attend College at Western Headquarters-UCR, during the \\ period from 2011-2012
}

\section{Doris Piñeiro Ruiz ${ }^{1}$}

Fecha de recepción: 6-6-2019

Fecha de aprobación: 26-8-2019

\begin{abstract}
Resumen
Este artículo es el resultado de una investigación cualitativa, cuyo fin es exponer los tipos de violencia por género que las estudiantes madres afrontan en diferentes escenarios, como lo es el familiar, educativo y de pareja, y algunas estrategias de empoderamiento que deben asumir para poder cumplir con su derecho a la educación superior. Sobre estos tipos de violencia menos visibles, más aceptadas y menos cuestionadas, se va a reflexionar, especialmente en: la violencia física, psicológica, sexual, económica e institucional, vividas por parte de las estudiantes universitarias madres en el contexto familiar, de pareja, e institucional. Para ello se trabajó con una población de ocho estudiantes madres que recibieron los servicios de la Casa Infantil Universitaria de la Sede de Occidente (CIUSO), de la Universidad de Costa Rica, durante el periodo 2011- 2012. De ahí que, se consideró importante traer a colación un tema que estaba siendo invisibilizado y ausente en las investigaciones anteriores efectuadas por el área de Ciencias Sociales, en esta Sede Universitaria.
\end{abstract}

Para ello, se utilizó el enfoque cualitativo, porque fue de vital importancia en el proceso de recolección y análisis de datos, ya que con este se pudo comprender las diversas estrategias de empoderamiento, tanto a nivel individual como colectivas que las estudiantes universitarias madres desarrollaron para enfrentar la situación de violencia y poder iniciar o reanudar sus estudios superiores.

Los resultados obtenidos mostraron la presencia de violencia de género en sus distintas manifestaciones, además, se determinó que en la población objeto de estudio estuvo presente tanto el empoderamiento social como el político, pues existía una mezcla de capacidades individuales, talento y voluntad en cada una de ellas y fue gracias a esos atributos personales que lograron esfuerzos colectivos.

Palabras claves: violencia de género hacia las mujeres, estrategias de empoderamiento, estudiante universitaria madre, educación superior

\footnotetext{
1 Licenciada en Trabajo Social, Magíster en el Estudio de la Violencia Social y Familiar, por la Universidad Estatal a Distancia. Docente e investigadora de la Carrera de Trabajo Social de la Universidad de Costa Rica, Sede de Occidente, Costa Rica. Departamento de Ciencias Sociales. Coordinadora de las prácticas institucionales de IV año de la Carrera de Trabajo Social -Sede de Occidente. Correo electrónico: dorispineiro@ hotmail.com- doris.pineiro@ucr.ac.cr
} 


\begin{abstract}
This article is the result of a qualitative research, with the purpose to analyze the types of violence by gender that Students as mothers face in different scenarios, such as family, educational and relationship context, and some empowerment strategies that they must assume to be able to fulfill their right to higher education.

On these less visible, more accepted and less questioned types of violence is what we are going to reflect on, more specifically: In the physical, psychological, sexual, economic, and institutional violence experienced by the Students as mothers, in the family, relationship, and institutional context. To this end, we worked with a population of eight students who are also mothers that received the services of the University Children's House of the Western Headquarters (CIUSO), of the University of Costa Rica, during the period 2011-2012. Hence, it was considered important to bring up a topic that was being made invisible and absent in previous researches conducted by the area of Social Sciences, at this University Headquarters. For this research the qualitative approach was used, because it was of vital importance in the process of gathering and analyzing the information, by using this method it was possible to understand the different empowerment strategies, both individually and collectively that the mothers that attend college developed to face these issues of violence and be able to start or resume their higher education.The results obtained showed the presence of gender violence in its different manifestations by which means it was possible to determine that the population under study had a strong presence of social and political empowerment, due to the fact that there was a mixture of individual capacities, talent and willpower in each one of them and it was thanks to those personal attributes that collective efforts were achieved.
\end{abstract}

Keywords: Gender violence towards women, Empowerment Strategies, Mother University Student, Higher education

\section{Introducción}

El presente artículo es el resultado de una investigación cualitativa para optar por el grado de maestría en el Estudio de la Violencia Familiar y Social, cuyo tema estuvo enfocado en analizar las "Estrategias de empoderamiento individuales y colectivas de las estudiantes universitarias madres que utilizaron el servicio de la Casa Infantil de la Sede de Occidente (CIUSO), Universidad de Costa Rica, frente a la violencia de género, para el acceso equitativo a la educación superior, como un derecho humano, en el periodo 2011-2012".

Para los fines de la investigación, se partió del concepto de violencia contra las mujeres enunciado por la ONU (1993, p.1), el cual refiere que es “(...) todo acto de violencia sexista que tiene como resultado posible o real un daño físico, sexual o psíquico, incluyendo las amenazas, la coerción o la privación arbitraria de libertad, tanto en la vida pública como en la privada". Por lo que, para darle un abordaje adecuado a esta problemática fue importante reconocer los diferentes escenarios donde las mujeres han sido violentadas y sus derechos irrespetados, teniendo claro que, los espacios son variados, pero el denominador común sigue siendo las relaciones desiguales de poder entre hombres y mujeres, reforzadas continuamente por el sistema patriarcal, en diferentes escenarios, como el familiar, educativo y de pareja, así como algunas estrategias de empoderamiento que asumieron durante su proceso de formación académica.

Para ello, se utilizó el enfoque cualitativo por considerarse de vital importancia en el proceso de recolección y análisis de la información, el cual es definido según Denzin y Lincoln (1994), citados por Vasilachis (2006, p.24) como aquel en donde "(...) se indagan situaciones naturales, intentando dar sentido o interpretar los fenómenos en los términos del significado que las personas le otorgan." Este enfoque se utilizó la intención de conocer algunas de las estrategias de empoderamiento que las estudiantes universitarias madres desarrollaron para enfrentar la situación de violencia y poder iniciar o reanudar sus estudios superiores.

En este sentido, se expondrán los tipos de violencia menos visibles y seguramente más aceptados y menos cuestionados, en torno a la violencia física, psicológica, sexual, económica e institucional, vividas por las 
estudiantes universitarias madres en el contexto familiar, de pareja e institucional, y de este modo, identificar algunas estrategias de empoderamiento que debieron fortalecer ante la violencia de género ejercida en su contra.

Dentro de este marco se ha visto, cómo a lo largo de la historia, los hombres han ocupado un lugar de dominio en espacios públicos, dominando autoritariamente los espacios privados, sobre todo el núcleo familiar. Este poder ha creado todo un sistema de jerarquías conocido como patriarcado, sistema en el que están incluidas todas las mujeres, independientemente de su condición.

Se trató de recabar la información a través de las propias narraciones de las mujeres involucradas en este estudio, enfocando la problemática de la violencia contra la mujer desde su perspectiva, pues se considera que la falta de equidad entre los géneros y la dominación masculina conforman la raíz principal del problema de violencia de género en nuestra sociedad.

Todos estos aspectos se consideran relevantes de exponer pues la mujer, como tal, debe cumplir una serie de normas y estereotipos sociales a costa de su satisfacción personal.

\section{Metodología}

En este apartado se expone la metodología seguida durante el proceso de investigación.

Se presenta el enfoque metodológico que guió el estudio, así como la población participante y los criterios bajo los cuales se establece la selección. Además, se contemplan las técnicas empleadas para la recolección de datos.

\subsection{Tipo de estudio}

Esta investigación tomó como punto de partida el enfoque cualitativo en el proceso de recolección y análisis datos, permitiendo tomar en cuenta las diversas formas de ver y sentir la realidad, siendo que todas las perspectivas de las estudiantes madres se consideren valiosas, sin importar si su punto de vista es antagónico al resto, al tiempo que trata de ser confiable a través de la exhaustividad (Gurdián, 2010).
Con este enfoque se procuró comprender las diversas estrategias de empoderamiento que las estudiantes universitarias madres desarrollaron para enfrentar la situación de violencia e iniciar o reanudar sus estudios superiores.

Desde lo expuesto por Mejía (2000, p.224), lo que interesó en este tipo de investigación fue: “(...) mirar de cerca el comportamiento de cada actor y el mundo de significados que produce”, de ahí que fue importante subrayar el significado que tenían las situaciones vividas por los sujetos desde su propia mirada y percepción.

En este sentido, el análisis realizado se basó en la categoría de género, tomando en cuenta la situación de violencia que enfrentaban estas estudiantes; la teoría de género se consideró pertinente al entender el problema en el marco de las relaciones de poder.

Es importante señalar que, al momento de plantear el problema, se descubre que este no había sido explorado a nivel local, lo que reflejó una escasez de material bibliográfico para profundizar en este. De ahí que tuvo un carácter exploratorio descriptivo, por estar dirigido a: “(...) explorar áreas, temas, hechos o situaciones sobre los que se ignora o existe escaso conocimiento acerca de sus causas y características" (Ramírez, Arcila, Buritica y Castrillón, 2004, p.22).

\section{2. Área de estudio}

El estudio se realizó en la Casa Infantil Universitaria (CIUSO), la cual se encuentra ubicada en el distrito de Alfaro de San Ramón de Alajuela, dentro de las instalaciones de la Sede de Occidente, Universidad de Costa Rica.

En el momento de realizar la investigación se contaba con una población de 25 estudiantes madres, las cuales procedían de distintas zonas geográficas del país, entre ellas: San Carlos, Esparza, Guanacaste, Guápiles, Naranjo, Grecia, San Ramón centro y distritos aledaños. 


\subsection{Población sujeta de estudio}

La población seleccionada fue de ocho estudiantes universitarias madres, cuatro que recibían los servicios de la CIUSO y cuatro que ya no formaban parte de esa población, porque sus hijos e hijas sobrepasaban la edad de los 4 años, pero que continuaban en el grupo de apoyo. Se consideró importante incluir a estas últimas porque habían contribuido a la dinámica grupal, siendo multiplicadoras del proceso de empoderamiento por asumir tareas de facilitación al grupo, así como participantes en la promoción de acciones contra la violencia de género e iniciativas de incidencia política, para el mejoramiento de las condiciones de estudio de las estudiantes madres, ante la toma de decisiones a nivel institucional.

\subsection{Criterios de selección}

- Que se encontraran incluidas en la lista de matrícula del primer y segundo ciclo lectivo del 20112012 en la Sede de Occidente, UCR

- Que recibieran los servicios de la CIUSO.

- Que hayan recibido los servicios de la CIUSO, y que por la edad de su hijo o hija (4 años) no se encontraran matriculadas en la misma, pero continuaban siendo estudiantes activas de la Sede de Occidente.

- Que las estudiantes madres seleccionadas hayan vivido una situación de violencia durante alguna relación de pareja, familiar o institucional, sin importar si esta es actual o pasada, ya que su experiencia facilitaría dar respuesta al tema de investigación planteado.

- Que expresaran disponibilidad para colaborar con la investigación.

- Tener como recurso físico la Cámara Gessell de la Sede de Occidente (SO) y/o la Casa Infantil Universitaria de la Sede de Occidente (CIUSO), para realizar con ellas las entrevistas, talleres y grupo focal, con el fin de ofrecer confidencialidad y comodidad a la hora de realizar las actividades citadas.
Las características particulares de este estudio fueron las siguientes:

Se desarrolló durante el periodo 2011- 2012.

- Se tomó como base el enfoque cualitativo, para tomar en cuenta las percepciones, opiniones y significados de una población de la sociedad como lo representan las mujeres madres, y en específico la realidad que viven cotidianamente aquellas que permanecen en el espacio universitario cuando están ejerciendo su maternidad.

- Fue de tipo exploratorio-descriptivo debido a que se buscaba ofrecer información valiosa sobre los tipos de violencia de género que sufren las estudiantes universitarias madres y las estrategias de empoderamiento desarrolladas tras enfrentar una situación de agresión.

Tomando en cuenta los aspectos mencionados, se tomó como referencia para su análisis las siguientes categorías:

- Tipos de violencia de género vividas por las estudiantes madres.

- Estrategias de empoderamiento.

- Acceso equitativo a la educación superior.

- Todos estos aspectos se consideraron relevantes en este estudio pues la mujer, como tal, debe cumplir una serie de normas y estereotipos sociales a costa de su satisfacción personal.

\subsection{Fuentes de información}

\section{Primarias}

- Estudiantes madres universitarias que recibieron los servicios de la CIUSO, UCR, cuyos hijos e hijas fueron cuidados (as) mientras recibían las lecciones.

- Personal encargado de la CIUSO, directora y docentes.

- Psicóloga de Vida Estudiantil de la SO. 


\section{Secundarias}

- Expedientes de las estudiantes madres de la CIUSO.

- Referencias bibliográficas (libros, tesis, artículos de revistas, entre otros).

\subsection{Técnicas de recolección de la información}

Se consideraron algunas técnicas cualitativas de investigación que permitieron indagar a profundidad la vida de las estudiantes madres, partiendo de sus percepciones, creencias y opiniones, como base fundamental de la información que se desea adquirir.

Se utilizó la entrevista semi estructurada, observación participante, grupos focales y la técnica de análisis de contenido. Esta última permitió analizar a profundidad los datos recabados, situando a la investigadora en una triple perspectiva, entre datos tal y como se le comunican, el contexto de dicha información y la manera cómo se interpreta esa realidad por parte de la autora.

Para el registro y transcripción de la aplicación de las entrevistas y del grupo focal, se contó con una grabadora de audio (con el consentimiento de la/s informantes), con el fin de dar validez la información y tener registro de prueba sobre la veracidad y confiabilidad de esta.

Estas grabaciones fueron transcritas por la investigadora para su interpretación final, para ello se seleccionó aquellos datos que respondiera al problema y objetivos de la investigación. No se utilizó ningún programa digital para el procesamiento de la información, en su lugar se elaboraron matrices, según las categorías y sub categorías de análisis para agrupar los datos de interés. Fue necesaria la utilización de seudónimos durante la codificación de los datos para referirse a las estudiantes universitarias madres, con el fin de garantizar la confidencialidad y validez del conocimiento obtenido. Para la elaboración de los seudónimos se eligieron nombres de flores con significados alusivos a las diferentes características que posee una persona con deseos de superación personal (perseverancia, confianza en sí misma, libertad, felicidad, valor en la adversidad, etc.). Antes de iniciar con cada entrevista, se les mostró una presentación en Power Point en la cual había imágenes de flores, con su significado respectivo, por lo que cada una tuvo la opción de elegir y asumir el nombre en el transcurso de la entrevista, para proteger su identidad.

\section{III.Marco Teórico}

Para realizar esta investigación fue necesario el estudio de diferentes teorías relacionadas con el tema y las categorías de análisis investigadas, las cuales se describen a continuación:

\subsection{Tipos de violencia de género contra la mujer en diferentes escenarios}

Para darle un abordaje adecuado a esta problemática, fue importante reconocer los diferentes escenarios donde las mujeres son violentadas y sus derechos irrespetados. Los espacios son variados pero el denominador común sigue siendo las relaciones desiguales de poder entre hombres y mujeres, reforzadas continuamente por el sistema patriarcal.

En este sentido, la violencia contra las mujeres se presenta bajo distintas modalidades y en diferentes espacios, para evidenciar esta afirmación se tomó como referencia el "Estudio a fondo sobre todas las formas de violencia contra la mujer”, (2006), realizado por Alméras y Calderón, el cual señala los siguientes escenarios:

a) Violencia dentro de la pareja: se manifiesta a través de actos sexuales, psicológica y físicamente coercitivos practicados contra mujeres adultas y adolescentes por una pareja actual o anterior, sin el consentimiento de ella. 
b) Violencia contra la mujer dentro de la familia: prácticas tradicionales nocivas: selección prenatal del sexo, matrimonio precoz, violencia relacionada con la dote, mutilación genital femenina, crímenes cometidos en nombre del "honor" y maltrato de viudas.

c) Feminicidio: homicidio de la mujer por razones de género

d) Violencia sexual infligida fuera de la pareja: actos perpetrados por parientes, amigos, conocidos, vecinos, compañeros de trabajo, de estudio o extraños. Incluye iniciación sexual forzada y violencia en citas.

e) Violencia contra la mujer en la comunidad, acoso sexual y violencia en el lugar de trabajo, en instituciones educativas o en espacios deportivos: acoso sexual o comportamiento sexual no deseado, explotación sexual y acoso por parte de entrenadores, espectadores, representantes, miembros de la familia o de la comunidad.

f) Violencia contra la mujer y discriminación múltiple: actores que pueden determinar discriminaciones múltiples: raza, origen étnico, casta, clase, condición de migrante, refugiada, edad, religión, orientación sexual, estado matrimonial, discapacidad, condición de portadora o no de VIH. (2012, pp.26-27).

Ante la situación expuesta, cualquier acción enfocada a combatir la violencia contra las mujeres (en todas sus manifestaciones) deberá tomar en cuenta estrategias que propicien la autonomía física, económica y política.

En consecuencia, se presentarán los tipos de violencia señalados de interés en este estudio, para dar respuesta a los objetivos propuestos.

\section{Violencia física}

Para conocer el grado de violencia física presente en las estudiantes madres se realizó una entrevista semi estructurada, enfocada en sus experiencias relacionadas con sucesos violentos. Se les preguntó si había sufrido, por parte de su pareja o antigua pareja, alguno de los eventos enunciados a continuación:

- Pegarle una cachetada.

- Lanzarle un objeto y herirla.

- Darle un empujón, jalarle el cabello o golpearle alguna parte de su cuerpo.

- Darle patadas hasta dejarla con moretones.

- Golpearla con algún objeto.

- Quemarla o rociarle algún líquido que pudiera dañarle la piel.

- Amenazarla con un arma o utilizar esta arma contra ella.

Las respuestas a estas preguntas fueron variadas, en realidad fueron muy pocas las estudiantes madres que manifestaron haber sido víctimas físicamente por parte de su pareja Sin embargo, lo relevante para el estudio es que aunque no todas las estudiantes fueron víctimas de violencia física, este es un acto que, aunque se dé una sola vez o se repita, puede ocasionar daños severos en la vida de la mujer, en donde su principal característica es el abuso del poder, utilizando la fuerza física del hombre.

\section{Violencia psicológica}

Este tipo de violencia provoca serios daños en la autoestima, pues la persona pierde la confianza en sí misma y aunque cree que es una persona libre, en realidad está siendo dominada. Esta situación la bloquea mentalmente impidiéndole razonar o comprender esa situación de maltrato de la cual es víctima. Como consecuencia de ese poder ejercido por el sexo masculino, la mujer no es capaz de defenderse del abuso, por el contrario, adquiere una actitud sumisa, al punto de proteger al 
agresor. Esta conducta en las estudiantes madres no es más que un reflejo del proceso de socialización en el cual han sido sometidas, proceso conocido como socialización patriarcal.

\section{Violencia sexual}

Esta sin duda es una de las expresiones más fuertes de la violencia hacia la mujer. El hombre la utiliza principalmente a la hora de tener relaciones sexuales, aunque su pareja no esté de acuerdo.

De todos los tipos de violencia expuestos, esta fue la menos mencionada por las estudiantes madres, no obstante, se debe tomar en cuenta la posible influencia del tabú que la sociedad le ha asignado a todo lo relacionado con el sexo y la sexualidad.

Por los daños emocionales y físicos que ocasiona este tipo de violencia también puede considerarse dentro del ámbito de invasión física o psicológica, porque sigue el patrón de reencauzar la conducta femenina y restablecer, tanto las reglas del poder que el hombre detenta, como la sumisión de la mujer.

\section{Violencia económica}

Se refiere a toda acción orientada al abuso económico y control de las finanzas de la mujer.

Vinculado a la definición anterior, las becas o subsidios económicos, otorgados por la Universidad, fueron de gran ayuda para aquellas estudiantes que provenían de familias con bajos ingresos, pues para muchas de ellas fue la principal fuente económica para cubrir los gastos de alimentación y de estudio durante su instancia académica, tomando en cuenta que sus parejas sentimentales no aportaban ninguna remuneración para hacerle frente a los gastos de ella y de su hijo(a).

Un dato importante de señalar es que, este subsidio económico en varias ocasiones fue utilizado para cubrir los gastos de la casa porque su compañero se encontraba desempleado y, ante la manipulación por parte de él, la estudiante se visualiza como la única persona que podía ayudarle. Una vez más, era víctima de los estereotipos de género, o mandatos sociales otorgados por la sociedad respecto al rol maternal que debe asumir en la familia.

\section{Violencia institucional}

Para Eternod, M. (2005) la violencia institucional tiene que ver con la "(...) violación de los derechos humanos desde las instituciones, es decir, los abusos u omisiones en las atribuciones y responsabilidades de las instituciones públicas" (p.26).

En efecto, Costa Rica, al igual que otros países, se ha preocupado por crear leyes para prevenir, sancionar y eliminar la violencia contra las mujeres. Sin embargo, no ha sido fácil lograr consensos para incluir la perspectiva de género en los lineamientos o protocolos institucionales, pues se requiere de mucha voluntad política para ponerse de acuerdo y caminar hacia la misma dirección.

Se sabe que la violencia institucional es el reflejo de la falta de interés de autoridades tanto públicas como privadas, cuyo accionar está respaldado por las mismas legislaciones, las cuales contienen muchos vacíos en materia de derechos humanos hacia las mujeres.

Este es un importante dato sobre la violencia institucional que pocas veces notamos y que, a pesar de ello, tiene consecuencias para el desarrollo humano y el acceso a los derechos.

\subsection{Estrategias de empoderamiento}

El concepto de empoderamiento es definido por la OPS y la OMS (2009, p. 27) como: “(...) la expansión de la libertad de escoger y de actuar. Significa aumentar la autoridad y el poder del individuo sobre los recursos y las decisiones que afectan su vida”. Asociados a este concepto están: “(...) la inclusión social, la autonomía, la visibilidad y movilidad, el agenciamiento (que es la capacidad de hacer uso de los activos de que dispone), y la autosuficiencia (que es la confianza en las competencias que le son propias a un individuo)" (OPS/OMS.2009, p. 27) 
Con el empoderamiento las mujeres lograrán asumir el control de sus propias vidas, pues contarían con la capacidad de elegir alternativas, tomar decisiones y ponerlas en práctica.

Se podría ver como un proceso, cuyo resultado traería beneficios a nivel colectivo e individual. También, se puede ver el empoderamiento de las mujeres como parte esencial en sus vidas, para el logro de la igualdad de género.

El poder solamente lo pueden adquirir ellas, nadie las puede empoderar. Sin embargo, las instituciones las pueden apoyar para reforzar o iniciar con el empoderamiento, tanto en el plano individual como colectivo (OPS/OMS: 2009).

Por su parte Casique (2010, p.1), señala que: "uno de los elementos que pueden ir asociados al empoderamiento de las mujeres es la disponibilidad de recursos (económicos y sociales) ... lo que, a su vez, el empoderamiento les da acceso a más y nuevos recursos".

Sin embargo, este concepto no se limita solamente a la adquisición de recursos (físicos, humanos, financieros), ideas, valores y actitudes, sino que va más allá de la toma de decisiones a nivel personal.

Por lo que hay que tomar en cuenta que los grupos sociales tienen diferencias, por razones de: etnia, edad o por género, y el tema del empoderamiento implica superar esas diferencias para crear estrategias en función de sus intereses.

Es así como, para los fines de esta investigación, se analizó el empoderamiento en dos dimensiones, a saber: social y político.

El empoderamiento social es un camino que lleva a varias direcciones en donde entran en juego el autoreconocimiento personal como sujetos portadores de derechos, el fortalecimiento del tejido institucional y el desarrollo de la capacidad de las organizaciones para incidir en los diferentes ámbitos de la vida, la economía, la política, y las instituciones (ASOCAM, 2007).
Este tipo de empoderamiento, guarda relación con la construcción de la identidad personal, la familia, la comunidad y las instituciones. También tiene que ver con la historia de vida de cada persona y la perspectiva de género que cada cual interioriza:

a) En lo social, a través del afianzamiento de valores y prácticas como la solidaridad, la colaboración, la autoconfianza y la promoción de la mujer.

b) En lo político por medio de una mayor participación en la toma de decisiones, la conciencia del interés individual y colectivo y el fomento de un sentido de responsabilidad y control social (ASOCAM, 2007).

Desde una visión política, el empoderamiento adquiere especial relevancia, pues la inclusión de los grupos vulnerabilizados, no solamente pasa por su fortalecimiento organizativo (empoderamiento social), sino también por una activa y deliberante participación en los espacios de poder y de decisión pública: "es un concepto sistémico, según el cual, cuando el poder de decisión y de control aumenta en aquellos que nunca lo tuvieron antes, el sistema se transforma (...) inevitablemente" (Pérez, 2005, citado por ASOCAM, 2007, p.14).

Entonces, se podría decir que, una mujer empoderada en términos políticos es aquella que ha tenido la capacidad de fortalecer sus valores, actitudes y aptitudes para lograr el éxito en diferentes espacios de diálogo y de decisión, analizando la realidad para crear propuestas de cambio ante una justa distribución en las relaciones de poder.

\subsection{Acceso a la Educación Superior como un derecho humano}

La educación de la mujer no se da en igualdad de circunstancias con respecto a la de los hombres, lo afirma Ana María Piussi (1997), citada por Moreno E, (sf, p.75): “(...) "la escuela de todos" ha sido en primer lugar escuela de los hombres". Anteriormente ellos eran los que tenían derecho a poseer el conocimiento, luego se incorporan las mujeres a la educación formal y esto se mantiene en aumento, gracias a la transformación que ha tenido la sociedad, aunque tiene mucho que ver los requerimientos de esta. Esta situación 
tendría que ser una causa esperanzadora de equidad, lo grave es que aún en este nivel, se torna desigual para los que lo conforman. Al llegar a este grado de estudios, las mujeres son vistas como privilegiadas, al igual que los hombres, porque su manera de conducirse y los trabajos a los que tiene acceso cambian; sin embargo, por su condición de estudiante y madre, ¿se podría decir que poseen las mismas oportunidades? La Unesco (1998, s-p), indica que "La 4 a Conferencia Mundial sobre la Mujer (Beijing, 1995) merece especial atención por haber marcado un hito en la historia de la emancipación social de la mujer y de su función en la dirección de la sociedad."

Se señala además que, muchos de los problemas que afrontan las mujeres enunciados en la Declaración de Beijing (1995) continúan sin resolverse, ejemplo de ello es:

- $\quad$ La pobreza.

- La falta de equidad en su acceso a la educación y la asistencia sanitaria y social.

- La violencia en sus distintas manifestaciones.

- Falta de equidad en su participación en las estructuras de poder de la sociedad.

- Insuficiente reconocimiento de sus derechos.

- Falta de reconocimiento de su contribución a la sociedad, entre otros.

Con el agravante de que estos problemas son un obstáculo para que las mujeres accedan a los diferentes espacios sociales y puedan asumir responsabilidades, como seres pensantes e importantes para el desarrollo de la sociedad. (UNESCO, 1998).

La situación se ha presentado de esta manera debido que, desde la historia, el papel de la mujer en la sociedad ha sido menospreciado y siempre se ha dicho que ellas deberían estar dentro de sus casas y nunca en los lugares públicos, se les ha considerado solamente como madres y ni siquiera como personas.

Lo que sí está claro es que el aporte de la mujer ha sido fundamental para el desarrollo de la sociedad, su presencia, al igual que los hombres, se ha sentido en diferentes campos, como la educación, ciencia, tecnología, entre otros.

Por ello, la educación representa la base principal para visibilizarla políticamente, pues una mujer educada, formada y preparada académicamente garantiza una sociedad justa, sin prejuicios de ninguna índole, de ahí que los planes estratégicos de las instituciones deben considerar la promoción de la educación femenina, con enfoque de género, en todos los ámbitos.

Por lo tanto, se puede concluir que, es urgente trabajar temas de violencia de género en el contexto universitario, para revertir la discriminación en contra de las madres estudiantes; pues es una situación que impacta en todos los niveles de la vida e impide que haya un ambiente adecuado para su desempeño académico.

\section{Reflexiones finales}

En este último apartado se expondrán las principales reflexiones, derivadas de las categorías de análisis desarrolladas durante el proceso investigativo.

Según los datos analizados las estudiantes madres han vivido algún tipo de violencia de género, ya sea por parte de su compañero sentimental, esposo, familiares cercanos o por docentes y compañero/as universitario/ as. Aunque no fueron todas las estudiantes madres quienes manifestaron haber sido víctimas de agresión física por parte de su pareja, esta estuvo presente en algunas de ellas, siendo el principal responsable su novio o compañero sentimental.

Es importante señalar que, existió resistencia para expresar la presencia de este tipo de violencia. Es posible que esto se deba a los mitos existentes alrededor de ella, pues aún hoy en día muchas mujeres consideran la vida familiar como "ámbito privado" y sienten que cuando comentan a alguien lo que les sucede, están traicionando a su pareja y a su "familia", porque hacen público lo que consideran privado. También, se debe a que es un tema que ha estado por muchos años silenciado, invisibilizado y normalizado, por lo que es común que se omita la información referente al hogar. 
Con respecto a la violencia psicológica, esta fue la más frecuente y fue ejercida principalmente por la familia y la pareja de la estudiante madre.

Fue interesante comprobar que, las familias no están exentas de reproducir en su interior el ejercicio de los poderes de dominio, por el contrario, son asignadas como una de las instituciones más efectivas para su reproducción.

Por su parte, la violencia institucional, se manifestó en el trato de varios/as docentes hacia las estudiantes madres, lo que provocó una revictimización hacia ellas.

También se debe señalar que la violencia institucional está presente cuando el contexto universitario no les facilita las condiciones mínimas de infraestructura para brindar los cuidados necesarios a sus hijos e hijas; ejemplo de ello son la falta de cambiadores en los baños, sala privada para amamantar, microondas accesible para calentar los alimentos, entre otras, estas condiciones no están presentes en todos los espacios de la Sede de Occidente.

Un dato importante de señalar es que, la mayoría de las estudiantes madres entrevistadas, y que fueron víctimas de violencia institucional, no la identificaron como tal, pues existía falta de reconocimiento de la violencia ejercida hacia ellas. Lo que trajo consigo una falta de denuncia de las situaciones vividas por miedo a no ser tomadas en serio o a no recibir apoyo por parte de las autoridades universitarias.

La violencia sexual, por su parte, se presentó en diferentes grados, desde las burlas y comentarios ofensivos, hasta comportamientos sexuales forzados mediante intimidaciones o presiones psíquicas, que obligaron a las estudiantes madres a tener sexo cuando no lo deseaban; el tipo más grave es la violació, aunque ellas no lo percibían en el momento se estaba presentando, cuando eran forzadas por sus compañeros sentimentales a mantener la relación sexual.

En cuanto a la violencia económica, fue la menos frecuente y estuvo presente en varias de ellas, debido a la falta de recursos económicos de sus compañeros para hacerle frente a los gastos de alimentación, por lo que debieron destinar parte del subsidio económico, aportado por la beca, para cubrir esa necesidad, igualmente las que se retiraron de sus casas en busca de mejores condiciones de convivencia familiar; sus compañeros hicieron uso de los muebles que habían adquirido entre ambos, para venderlos y obtener ganancias económicas y no compartirlo con ellas.

Con respeto a la segunda categoría de análisis estudiada, se logra determinar que, las estrategias de empoderamiento en las estudiantes universitarias madres estuvo presente, tanto el empoderamiento social como el político, pues existía una mezcla de capacidades individuales, talento, voluntad e inteligencia en cada una de ellas, gracias a los atributos personales que lograron a través de los esfuerzos colectivos, un empoderamiento en donde esta fue capaz de expresar con diálogo su pensamiento, con ideas claras, convincentes y con respeto hacia los demás.

Una mujer empoderada políticamente se reconoce como aquella que accede con éxito a espacios de debate, sin imponer sus ideas y promueve el cambio en las relaciones del poder.

Para finalizar con la tercera categoría analizada, “Acceso equitativo a la educación superior", se confirmó que, aunque el sistema de educación no reproduce los estereotipos, sí los mantiene y refuerza, es decir, que define y redefine los géneros. Uno de los aspectos más sobresalientes en el análisis de las respuestas, fue la clara manifestación de valoraciones que el cuerpo docente tiene, de lo que es ser un hombre y ser mujer, así como los papeles asignados a cada uno de ellos; los ven como naturales dentro de la sociedad patriarcal.

Las estudiantes madres participantes de la investigación tuvieron un proceso de socialización marcado por el ideario patriarcal y fueron afectadas por esta sociedad androcéntrica, vivenciado formas de violencia (física, psicológica, sexual, económica e institucional) que son producto de las diferencias genéricas que la sociedad 
ha establecido para hombres y mujeres, al igual que discriminaciones, donde se les ha negado, entre varios elementos, el acceso equitativo a la educación superior, como un derecho humano.

Como complemento, se retoman las palabras de Ferrer (2012) la cual indica que:

(...) trabajar el empoderamiento de las mujeres conlleva trabajar desde una perspectiva de género, desde donde se fomente la igualdad entre hombres y mujeres y se les capacite para ser protagonistas de su propia vida, disfrutando de una plena libertad de elección y expresión, con base a un pensamiento crítico, para que puedan llegar a ser todo lo que quieren sin necesidad de depender de nada ni de nadie (p.8).

En ese sentido, la violencia de género contra la mujer solamente se puede erradicar eliminando todo tipo de discriminación, en donde el empoderamiento sea la base principal para el ejercicio de los derechos humanos.

Por último, es importante señalar que, por ser la primera investigación relacionada con la violencia de género en estudiantes universitarias madres, en la Sede de Occidente de Universidad de Costa Rica, no se tuvo ningún impedimento por parte de la población sujeta de estudio para brindar la información, al contrario, se convirtió en una oportunidad para hacer un llamado a las autoridades universitarias para tomar acciones, solicitando la erradicación de estereotipos de género, sesgos sexistas y diversas formas de discriminación en los procesos de socialización y educación que limitan tanto las oportunidades como capacidades de estudiantes madres y padres.

\section{Bibliografía}

Alméras, D y Calderón Magaña, C. (2012). Si no se cuenta, no cuenta. Información sobre la violencia contra las mujeres., Santiago, Chile. [En línea] Santiago [Citado 21-04-12]Disponible en internet: https://repositorio.cepal.org/bitstream/ handle/11362/27859/S2012012_es.pdf

ASOCAM (2007). "Reflexiones y Aprendizajes" Empoderamiento: conceptos y orientaciones. Quito, .[En línea] [citado 16-04-12] Disponible en internet: https://www.shareweb.ch/site/ 2007.pdf

Casique. I. (2010). Factores de empoderamiento y protección de las mujeres contra la violencia. En Revista Mexicana de sociología. vol.72 no.1 México ene./mar. 2010. [En línea] México [Citado 21-04-12]Disponible en internet: http:// www.scielo.org.mx/scielo.php?script $=$ sci arttext\&pid=So188-25032010000100002

ENDIREH. (2003). Encuesta Nacional sobre la Dinámica de las Relaciones en los Hogares. INEGI, México. [En línea] México [Citado 21-04-12]Disponible en internet: http://cedoc.inmujeres.gob.mx/ documentos_download/100492.pdf

Eternod, M. (2005.) El Sistema de estadísticas sobre violencia en el Instituto de Estadística, Geografía e Informática. (p.26). En Instituto Nacional de las Mujeres. (2005). Reunión internacional de atención y prevención de la violencia hacia las mujeres: Un enfoque multidisciplinario. México. [En línea] [Citado 20-06-12] Disponible en internet: http:// www.inmujeres.gob.mx

Ferrer Lanchares. Y. (2012). Intervención con grupos de mujeres para el empoderamiento y la prevención de la violencia de género en la provincia de Palencia. Grado de Educación social. E.U.E de Palencia. Universidad de Valladolid. [En línea] [Citado 2006-12] Disponible en internet: http://uvadoc.uva.es/ bitstream/handle/10324/2076/tfg-;jsessionid=A19E AgD3BoC18AB23E826DC49834543F?sequence=1 
Gurdián, A. (2010). El paradigma cualitativo en la investigación socio-educativa. San José, Costa Rica: Editorial Universidad de Costa Rica.

Mejía. Julio. (2000). "Técnicas cualitativas de investigación en ciencias sociales". Revista: Investigación Educativa. Año 4, No. 7. Universidad Nacional Mayor de San Marcos.Lima, Perú.

Moreno, E. (sf). La transmisión de modelos sexistas en la escuela. Sin más referencia. [En línea] [Citado 21-04-12]Disponible en internet: https://www. dgespe.sep.gob.mx/public/genero/PDF/GEN\%20 O1/G_01_02_La $\% 20 t$ ransmisi $\% C_{3} \%$ B3n $\% 20$ de\%2omodelos.pdf

ONU (1993). Declaración sobre la eliminación de la violencia contra la mujer. Art. 1. 85 a sesión plenaria, 20 de diciembre de 1993. [En línea] [Citado 20-06-12] Disponible en internet: https:// www.ohchr.org/sp/professionalinterest/pages/ violenceagainstwomen.aspx

OPS/OMS. (2009). Empoderamiento de mujeres adolescentes. Una publicación producida por el Área de Salud Familiar y Comunitaria (FCH) de la Organización Panamericana de la salud / Organización Mundial de la Salud. [En línea] Washington. D.C. [citado 21-04-12] Disponible en internet: http://new.paho.org/ hq/dmdocuments/2010/Empoderamiento\%20 de\%2oMujeres\%2oAdolescentes.pdf

Ramírez Robledo L, E, Arcila, A, Buriticá, L, E y Castrilló, J. (2004). Paradigmas y Modelos de Investigación. Guía Didáctica y Módulo. Fundación Universitaria Luis Amigó. Facultad de Educación. 2 edición. [En línea] [Citado el 20-08-12 Disponible en internet http://virtual. funlam.edu.co/repositorio/sites/default/files/ repositorioarchivos/2011/02/ooo8parad igmasymodelos.771.pdf
UNESCO. (1998). Declaración Mundial sobre la Educación Superior en el Siglo XXI: visión y acción y Marco de Acción prioritaria para el cambio y el Desarrollo de la Educación Superior. Conferencia Mundial sobre la Educación Superior. [En línea] [Citado 21-04-12- y el 13-04-13] Disponible en: : http://www.unesco.org/education/educprog/ wche/declaration_spa.htm

UNESCO. (1998). Mujeres y educación superior, cuestiones y perspectiva.[En línea] [Citado el 12-04-13]. Disponible en: http://www.unesco.org/education/ educprog/wche/principal/women-s.html

Vasilachis, I. (2006). Estrategias de investigación cualitativa. Barcelona, España: Editorial.GEDISA. 